Dicenda. Estudios de lengua y literatura españolas

ISSN-e: 1988-2556

https://dx.doi.org/10.5209/DICE.65006

\title{
Análisis de la influencia de Charles Dickens en el estilo de Benito Pérez Galdós a través del lenguaje gestual de sus personajes: un estudio de corpus
}

\author{
Guadalupe Nieto Caballero ${ }^{1}$
}

Recibido: 31 de julio de 2017 / Aceptado: 27 de noviembre de 2018

Resumen. En este artículo se analiza, a través de un enfoque de estilística de corpus, la influencia que Charles Dickens ejerció en el estilo de Benito Pérez Galdós. El estudio se ha centrado en el lenguaje gestual de los personajes. De forma más concreta, se han analizado aquellos clusters empleados de forma recurrente por ambos autores que contienen referencias a alguna de las siguientes partes del cuerpo: los ojos, la cabeza, las manos o los hombros. Como se podrá comprobar, Dickens y Galdós utilizan construcciones similares - tanto en términos formales como funcionales- para construir sus universos ficticios, lo que ayudará a demostrar la influencia del primero sobre el segundo desde un punto de vista estilístico.

Palabras clave: Galdós; Dickens; estilística de corpus; influencia.

\section{[en] A corpus-based approach to Charles Dickens's alleged influence on Benito Pérez Galdós through the use of their characters' body language}

\begin{abstract}
In this article, Charles Dickens's alleged influence on Benito Pérez Galdós's style is analysed from a corpus-stylistic point of view. The study has concentrated on body language. More specifically, a series of clusters employed by both authors containing one of the following four body parts have been scrutinised: eye, hand, head and shoulder. As will be shown, both authors make an almost analogous use of similar clusters to build their fictive universes, which will help to demonstrate that Dickens's influence in Galdós goes well beyond the impressionistic references traditionally discussed, having a purely stylistic basis hitherto underexplored.
\end{abstract}

Keywords: Galdós; Dickens; corpus stylistics; influence.

Sumario: 1. Introducción; 2. La influencia de Dickens en Galdós: 3. Metodología; 4. Resultados; 5. Análisis; 5.1 "Ojo" - eye; 5.2 "Mano" — hand; 5.3 "Cabeza" — head; 5.4 "Hombro" — shoulder; 6. Apuntes finales.

Cómo citar: Nieto Caballero, G. (2019). Análisis de la influencia de Charles Dickens en el estilo de Benito Pérez Galdós a través del lenguaje gestual de sus personajes: un estudio de corpus, en Dicenda. Estudios de Lengua y Literatura Españolas, 37, 321-341.

Universidad de Extremadura

gnieto@unex.es 


\section{Introducción}

Una de las mayores ventajas de los estudios de estilística de corpus reside en su capacidad para combinar enfoques de crítica literaria tradicional con otros más novedosos propios de la lingüística de corpus, lo que permite la identificación de patrones textuales estilísticamente relevantes que tradicionalmente han pasado desapercibidos en el análisis literario de la obra de un autor determinado (Mahlberg, 2013: 27). El potencial de este tipo de análisis queda patente en los estudios realizados sobre los autores más importantes en lengua inglesa ${ }^{2}$, así como en estudios comparativos sobre el estilo de estos ${ }^{3}$. Este potencial contrasta, sin embargo, con la ausencia de trabajos sobre autores en lengua española, en donde la estilística de corpus constituye una disciplina aún por explotar. Este artículo pretende demostrar, a través de la figura de Benito Pérez Galdós, la validez de estos análisis en autores en lengua española. De forma más concreta, en este estudio se analiza, utilizando un enfoque de corpus, la influencia que Charles Dickens ejerció en el estilo del autor canario. Para ello, se abordará la producción narrativa de ambos autores. El análisis se centra en el lenguaje gestual, un sistema autónomo en la construcción del universo ficticio en el género novelesco en general (Korte, 1997: 4) y uno de los rasgos distintivos del estilo de Dickens en particular (Mahlberg, 2013: 100-127). El estudio gira en torno a segmentos de texto de al menos cinco palabras empleados de forma sistemática por ambos novelistas que hagan referencia alguna de las siguientes partes del cuerpo: los ojos, la cabeza, las manos o los hombros. Por ejemplo:

(1) 'It happens very fortunately, Varden, 'said his wife, with her handkerchief to her eyes, 'that in case any more disturbances should happen.'

(Barnaby Rudge, capítulo 51) ${ }^{4}$

(2) La despedida fue tiernísima. María Cristina tan pronto se llevaba el pañuelo a los ojos, como saludaba a la multitud agitándolo, sin poder decir más palabra que adiós, adiós...

(Montes de Oca, capítulo 9)

Tanto her handkerchief to her eyes como el pañuelo a los ojos, como se detallará en el apartado 5.1, no solo son construcciones similares utilizadas de forma recurrente por Dickens y Galdós respectivamente, sino que además desempeñan una función estilística similar en la producción de ambos autores: las dos se asocian al habla de personajes femeninos y, además, son empleadas en momentos dialógicos para reforzar la tristeza de estos. La amplia variedad de ejemplos localizados en las novelas de ambos escritores, así como las similitudes estilísticas en el uso que tanto Dickens como Galdós hacen de ellos, revelan un eco dickensiano en el estilo del autor canario

2 Algunos autores cuyo estilo ha sido abordado desde esta perspectiva son: Virginia Woolf (Adolphs y Carter, 2002), Conrad (Stubbs, 2005), Shakespeare (Culpeper, 2009), Austen (Fischer-Starcke, 2010) o el propio Dickens (Mahlberg, 2013; Ruano San Segundo, 2016).

3 Sirva como ejemplo el análisis comparativo de Ruano San Segundo (2017) sobre la producción narrativa del propio Dickens y George Bernard Shaw, en donde se analiza la influencia del primero sobre el segundo desde un punto de vista estilístico.

4 Todos los ejemplos citados en el artículo han sido extraídos de una versión electrónica de los textos (véase apartado 3). Por lo tanto, en lugar de la paginación, únicamente se ofrece como referencia el capítulo en el que aparecen. 
que servirá para reforzar la consabida influencia que sobre él ejerció Charles Dickens y cómo ambos utilizan fórmulas de época similares ligadas los estereotipos que se manejan en el siglo XIX europeo 5 .

El artículo se organiza como sigue. En primer lugar, se hace un repaso general de los aspectos en los que se tradicionalmente se sustenta la influencia de Dickens en Galdós (apartado 2). A continuación, se detalla la metodología que se ha empleado para localizar los ejemplos que posteriormente se analizan (apartado 3). En tercer lugar, se muestran los resultados obtenidos en cada uno de los corpus de estudio con las distintas partes del cuerpo objeto de análisis (apartado 4). Finalmente, se analiza una selección de ejemplos (apartado 5), que servirán para afianzar la influencia de Dickens en el estilo de Galdós a través de aspectos concretos que hasta ahora han pasado desapercibidos para la crítica literaria. El artículo finaliza con una reflexión en torno al potencial de los estudios de corpus en el análisis de textos literarios y algunas sugerencias para futuros estudios sobre la obra de Galdós.

\section{La influencia de Dickens en Galdós}

Es de sobra conocida la influencia que grandes literatos como Cervantes (Goldman, 1971; Benítez, 1990), Balzac (Lacosta, 1968; Ollero, 1973) y el propio Dickens (McGovern, 2000; Tambling, 2013) ejercieron sobre Galdós. Así lo reconoció el autor canario, quien en Memorias de un desmemoriado, por ejemplo, aseguraba que consideraba "a Carlos Dickens como mi maestro más amado. En mi aprendizaje literario, cuando no había salido yo de mi mocedad petulante, apenas devorada $L a$ Comedia humana de Balzac, me apliqué con loco afán a la copiosa obra de Dickens" (Pérez Galdós, 1980: 1693). En ese aprendizaje literario se sitúa precisamente su traducción de Pickwick Papers para el diario madrileño La Nación, que sirvió no solo de homenaje al autor victoriano, sino también para absorber el estilo de uno de sus referentes (Wright, 1979: 24). Tradujo el Pickwick en 1868, cuando apenas tenía veinticuatro años y mientras se encontraba inmerso en la redacción de su primera novela, La Fontana de Oro, publicada un par de años más tarde. Tal vez por ello en La Fontana se advierten ecos dickensianos fácilmente reconocibles, como las descripciones minuciosas de la apariencia física de los personajes, la postura jocosa del narrador en determinados momentos de la historia o su animadversión a los opresores.

El de La Fontana de Oro es solo un ejemplo paradigmático de los numerosos rastros del autor inglés en la obra de Galdós en los que se sustenta la influencia apuntada por la crítica. Conviene destacar, sin embargo, que esta influencia se basa sobre todo en referencias impresionistas más que en análisis de tipo estilístico. Dicho de otro modo, aunque la influencia de Dickens en Galdós es un hecho incontrovertible y nadie duda de que la prosa dickensiana permeó su estilo, no existen estudios sistemáticos de tipo estilístico que analicen tal influjo. Por el contrario, la crítica apenas suele hacerse eco de aspectos muy llamativos del estilo dickensiano que gozan de réplica en la obra de Galdós, como el uso los nombres simbólicos y las muletillas

\footnotetext{
Para una información más detallada al respecto del uso de estereotipos en términos de caracterización, consúltense los trabajos de Correa (1967) sobre la figura de Galdós y los de John (2001) y Ruano San Segundo (2018) sobre la figura de Dickens.
} 
que emplean algunos personajes para caracterizarlos. En el caso de los nombres, pueden señalarse, a modo de ejemplo, casos como Doña Perfecta o Don Inocencio en Doña Perfecta, similares a Charity Pecksniff, Mercy Pecksniff o Mr. Wolf en Martin Chuzzlewit. En cuanto a las muletillas, por otro lado, pueden mencionarse en toda la extensión de la palabra, de Doña Lupe (Fortunata y Jacinta), o y ole morena y digo más, de Villaamil (Miau), con una función caracterizadora similar a la de not to put too fine a point upon it, de Mr. Snagsby (Bleak House) o I'll eat my head, de Mr. Grimwig (Oliver Twist). Asimismo, la crítica suele acompañar estos aspectos con analogías de contenido para reforzar la influencia de Dickens en el estilo del autor canario ${ }^{6}$. Estas analogías suelen ser fundamentalmente de dos tipos: de situaciones en las historias de Galdós que se asemejan a otras de las novelas de Dickens y, sobre todo, de personajes similares a los que pueblan el universo dickensiano. En el caso de las primeras, puede señalarse la escuela de don Pedro Polo en El doctor Centeno, caracterizada por unos métodos feroces que evocan a los de las escuelas que nos plantea Dickens en algunas de sus novelas, como Dotheboys Hall en Nicholas Nickleby, regida por Squeers con una brutalidad despiadada, o Salem House en David Copperfield, administrada por Mr. Creakle con métodos igualmente crueles. En cuanto a los personajes, los ecos dickensianos son de muy diversa índole. En ocasiones, tienen que ver con la apariencia física, como el caso de Marianela (Marianela), cuya diminuta estatura se asemeja a la de Amy Dorrit (Little Dorrit) o la pequeña Nell (The Old Curiosity Shop), lo que contribuye a presentarlas como figuras indefensas (McGovern, 2000: 139). Otras veces, el paralelismo se advierte en las relaciones que se establecen con otros personajes de la novela, como en el caso de los usureros Pepet (La loca de la casa) y Daniel Quilp (The Old Curiosity Shop), cuya imagen se ve reforzada por la contraposición que los autores hacen de ellos en relación a las figuras angelicales de Victoria y la pequeña Nell respectivamente. Por último, las similitudes más llamativas se advierten en aquellos personajes galdosianos que parecen un calco de alguna figura del autor victoriano. Tal es el caso, por ejemplo, de Torquemada en la tetralogía de las novelas de Torquemada, muy similar a Paul Dombey en Dombey and Son: ambos están casados con mujeres hermosas de familias acomodadas, ignoran a sus hijas a pesar del amor que estas muestran por sus padres, la acumulación de riquezas es el fin último de ambos y son dueños de una fortuna de la que finalmente pierden el control.

Todos estos ejemplos, sin duda, ilustran la influencia que Dickens ejerció sobre Galdós, si bien es cierto, como se decía anteriormente, que se trata de referencias de tipo impresionista más que de similitudes estilísticas como tal. Así pues, el hecho de que comúnmente se haya aceptado la influencia de Dickens en el estilo de Galdós basándose en una compilación de referencias dickensianas como las que acaban de detallarse no solo justifica, sino parece demandar una aproximación sistemática al estilo de ambos autores. Solo de esta manera será posible calibrar en qué medida el estilo de Dickens influyó en el de Galdós más allá del uso de nombres simbólicos y el empleo de muletillas por parte de sus personajes comentados más arriba. En este artículo se analiza esa influencia a través del ejemplo del lenguaje gestual mediante un enfoque de corpus. Como se verá, ambos autores hacen un uso similar de las mismas construcciones para modelar sus universos ficticios. La recurrencia de estas construcciones, así como sus similitudes tanto en términos formales como funcio-

Véanse, por ejemplo, Levy (1937), McGovern (2000) o Tambling (2013). 
nales, servirán para demostrar una concomitancia entre ambos que hasta ahora ha pasado desapercibida, lo que reforzará una influencia que, si bien fuera de toda duda, no se ha sustentado sobre una base lo suficientemente sólida desde una perspectiva puramente estilística.

\section{Metodología}

Los textos que forman ambos corpus de estudio se han recopilado de dos repositorios digitales distintos. En el caso del corpus de Galdós (en adelante, CorBPG), los textos han sido descargados de la Biblioteca Virtual Miguel de Cervantes. Este repositorio, que tiene como objetivo la difusión de la cultura hispánica en general, cuenta con alrededor de doscientos mil registros bibliográficos, de los cuales sesenta mil son libros. La calidad de la versión digitalizada de los textos, fundamental en los estudios de corpus como el que aquí se realiza, está fuera de toda duda, pues la Biblioteca cuenta con un consejo científico que avala el rigor de los materiales alojados en el repositorio. En el caso de Galdós, toda su producción literaria se encuentra digitalizada. Para llevar a cabo este análisis, se ha seleccionado su obra narrativa, formada por ochenta y cinco títulos y con una extensión de más de seis millones de palabras, como se muestra en la tabla $1^{7}$. Sus obras de teatro no han sido incluidas en el corpus por no pertenecer al género narrativo.

Tabla 1. Obras incluidas en CorBPG.

\begin{tabular}{|l|r|}
\hline Narrativa de Galdós & Palabras \\
\hline Episodios Nacionales (primera serie) & 685566 \\
\hline Episodios Nacionales (segunda serie) & 640781 \\
\hline Episodios Nacionales (tercera serie) & 759432 \\
\hline Episodios Nacionales (cuarta serie) & 774084 \\
\hline Episodios Nacionales (quinta serie) & 431931 \\
\hline Novelas (serie primera época) & 683209 \\
\hline Novelas (serie contemporánea) & 2042569 \\
\hline Narrativa Breve & 17059 \\
\hline Total & 6034631 \\
\hline
\end{tabular}

El corpus de Dickens (en adelante, CorCD), por otro lado, está formado por textos descargados de Proyecto Gutenberg. Este repositorio digital está formado por más de cincuenta mil libros de dominio público convertidos en archivos de texto electrónicos. También se encuentran disponibles de forma gratuita. Aunque existen

Los ochenta y cinco títulos que conforman CorBPG pueden consultarse en la página web de la Biblioteca Virtual Miguel de Cervantes: http://www.cervantesvirtual.com/bib/bib_autor/galdos/obra.shtml. 
voces reticentes en torno al uso de este material en el ámbito de la investigación científica por tratarse de textos compilados a raíz de contribuciones de voluntarios (véase Berglund, Morrison, Wilson y Wynne, 2004: 14), el amplio número de estudios realizados con material obtenido de este repositorio (véanse, por ejemplo, Barnbrook, 1996; Manning y Schütze, 1999; o Römer, 2006) avala la calidad de los textos. Asimismo, cabe destacar que los estudios de estilística de corpus en torno a la figura de Dickens realizados hasta la fecha también han sido realizados con textos de Proyecto Gutenberg (Mahlberg, 2007; Mahlberg, 2013; Mahlberg y Smith, 2012; Mahlberg, Smith y Preston, 2013; Ruano San Segundo, 2016, entre otros), lo que refuerza su validez. En el caso concreto de este análisis, se han seleccionado las catorce novelas completas del autor, con una extensión de casi cuatro millones de palabras. Estas se muestran en la tabla 2:

Tabla 2. Obras incluidas en CorCD.

\begin{tabular}{|l|c|}
\hline Novelas & Palabras \\
\hline Pickwick Papers & 307401 \\
\hline Oliver Twist & 160308 \\
\hline Nicholas Nickleby & 329201 \\
\hline The Old Curiosity Shop & 220612 \\
\hline Barnaby Rudge & 258569 \\
\hline Martin Chuzzlewit & 343316 \\
\hline Dombey and Son & 361766 \\
\hline David Copperfield & 361761 \\
\hline Bleak House & 360181 \\
\hline Hard Times & 105071 \\
\hline Little Dorrit & 343459 \\
\hline A Tale of Two Cities & 137503 \\
\hline Great Expectations & 187544 \\
\hline Our Mutual Friend & 332343 \\
\hline Total & 3809387 \\
\hline
\end{tabular}

Para procesar los textos que conforman ambos corpus se ha empleado el software de concordancias WordSmith Tools 7 (Scott, 2016a). El análisis, como ya se ha comentado, gira en torno a grupos de palabras (clusters) utilizados de forma recurrente por ambos autores en los que se haga referencia a alguna de las siguientes partes del cuerpo: los ojos, la cabeza, las manos o los hombros. Naturalmente, ni estas partes del cuerpo ni los ejemplos que se han analizado han sido escogidos de forma arbitraria. En el caso de las partes del cuerpo, por un lado, se han seleccionado estas 
cuatro por su relevancia estilística en la obra del autor inglés ${ }^{8}$. Dado su valor en las novelas de Dickens, estas partes del cuerpo resultan pertinentes como elemento de comparación que permita calibrar la influencia del autor victoriano en Galdós desde un punto de vista estilístico a través del lenguaje gestual. Por otro lado, los ejemplos que contienen alguna de estas cuatro partes del cuerpo que se han analizado han sido generados de forma automática con WordSmith Tools. El hecho de que los ejemplos no hayan sido seleccionados de forma premeditada contribuye, sin duda, a reforzar la validez de las conclusiones extraídas. En concreto, se han analizado aquellos grupos de palabras que, además de hacer referencia a al menos una de las cuatro partes del cuerpo que se han mencionado, tengan una longitud mínima de cinco palabras y una frecuencia de al menos diez apariciones en cada corpus.

En cuanto a la localización de los clusters, como se sabe, estos se generan sobre una lista de palabras (index) creada previamente con los textos que conforman cada corpus ${ }^{9}$. Al ser generados automáticamente, WordSmith no solo identifica aquellos que contengan alguna de las cinco partes del cuerpo que aquí se analizan, sino todos los grupos de palabras que se encuentren dentro de los parámetros establecidos. En la ilustración 1, por ejemplo, se muestran los veinte clusters más numerosos en CorBPG con una longitud mínima de cinco palabras.

\begin{tabular}{|c|c|c|c|c|c|c|c|c|c|}
\hline \multicolumn{6}{|c|}{ W index_BPG_file_index_5-7-word clusters.Ist } & \multirow{2}{*}{\multicolumn{2}{|c|}{$-\quad \square$}} & \multirow{2}{*}{\multicolumn{2}{|c|}{$\times$}} \\
\hline File É Edit & View Compute & Settings Windows Help & & & & & & & \\
\hline N & & Word & Freq. & $\%$ & Texts & & Lemmas & Set & $\hat{\imath}$ \\
\hline 1 & & A UN LADO Y OTRO & 118 & & 50 & 56,18 & & & \\
\hline 2 & & DE LA CALLE DE LA & 111 & & 39 & 43,82 & & & \\
\hline 3 & & EN LA CALLE DE LA & 89 & & 43 & 48,31 & & & \\
\hline 4 & & EN LA PUERTA DE LA & 83 & & 42 & 47,19 & & & \\
\hline 5 & & EN LA CUENTA DE QUE & 73 & & 44 & 49,44 & & & \\
\hline 6 & & A LA PUERTA DE LA & 64 & & 35 & 39,33 & & & \\
\hline 7 & & CASA DE LA CALLE DE & 64 & & 33 & 37,08 & & & \\
\hline 8 & & EN MEDIO DE LA CALLE & 63 & & 34 & 38,20 & & & \\
\hline 9 & & EN LA PUERTA DEL SOL & 59 & & 27 & 30,34 & & & \\
\hline 10 & & DE UN LADO PARA OTRO & 57 & & 35 & 39,33 & & & \\
\hline 11 & & EN EL MOMENTO EN QUE & 57 & & 33 & 37,08 & & & \\
\hline 12 & & LA PUERTA DE LA CASA & 56 & & 32 & 35,96 & & & \\
\hline 13 & & LAS MANOS A LA CABEZA & 56 & & 35 & 39,33 & & & \\
\hline 14 & & LA ESQUINA DE LA CALLE & 55 & & 33 & 37,08 & & & \\
\hline 15 & & YONO SÉ LO QUE & 54 & & 37 & 41,57 & & & \\
\hline 16 & & DE UNA PARTE A OTRA & 52 & & 30 & 33,71 & & & \\
\hline 17 & & NO HAY MÁS REMEDIO QUE & 52 & & 29 & 32,58 & & & \\
\hline 18 & & NO ES MÁS QUE UN & 50 & & 36 & 40,45 & & & \\
\hline 19 & & NO HABIA MÁS REMEDIO QUE & 50 & & 28 & 31,46 & & & \\
\hline 20 & & POR LA CALLE DE LA & 48 & & 26 & 29,21 & & & $\checkmark$ \\
\hline$<$ & & & & & & & & $>$ & \\
\hline frequency & alphabetical & filenames notes & & & & & & & \\
\hline 5.905 entri & Row 13 & \begin{tabular}{l|l}
$\mathrm{T}$ & $\mathrm{S}$ \\
\end{tabular} & & MANC & SSALA & CABEZA & & & \\
\hline
\end{tabular}

Ilustración 1. Clusters más recurrentes en CorBPG.

8 Para un análisis de la función de estas partes del cuerpo en las novelas de Dickens, véase Mahlberg (2013), el estudio más completo sobre el uso del lenguaje gestual en la producción del autor inglés.

9 Para una información más detallada acerca de cómo generar clusters con WordSmith Tools, véase Scott (2016b). 
Sobre la lista de clusters que se genera con cada uno de los dos corpus de estudio, es necesaria una búsqueda para localizar aquellos ejemplos que contengan alguna de las cuatro partes del cuerpo que se han comentado - la opción Wordlist permite hacer pesquisas concretas a través de una caja de búsqueda. En la ilustración 1, por ejemplo, vemos que las manos a la cabeza es, con cincuenta y seis apariciones, el cluster más recurrente en la producción de Galdós que contiene alguna de las partes del cuerpo que aquí se analizan. A continuación se muestran los resultados que se han obtenido con cada uno de los corpus, que constituyen el material con el que se ha llevado a cabo el análisis.

\section{Resultados}

Los resultados han sido clasificados según las cuatro partes del cuerpo que acaban de comentarse. En este sentido, conviene señalar que aquellos clusters que contienen referencias a dos partes del cuerpo (las manos a la cabeza, por ejemplo) han sido clasificados según la parte del cuerpo que parece definir mejor el movimiento que expresan. Así, el mencionado ejemplo de las manos a la cabeza ha sido incluido dentro del grupo de clusters que contienen la palabra "cabeza". Esta decisión, que por lo demás no acarrea ninguna implicación que pudiera influir en el análisis que aquí se lleva a cabo, se ha tomado para evitar duplicidades en los ejemplos que se muestran a continuación.

En primer lugar, en la tabla 3 se muestran los ejemplos localizados en ambos corpus que contienen la palabra "ojo" y eye:

Tabla 3. Clusters que contienen la palabra eye u "ojo".

\begin{tabular}{|l|c|l|c|}
\hline CorBPG & Frec. & CorCD & Frec. \\
\hline con los ojos fijos en & 22 & his eyes fixed on the & 17 \\
\hline echando lumbre por los ojos & 21 & with his eyes fixed on & 16 \\
\hline abrir y cerrar de ojos & 17 & with his eyes on the & 13 \\
\hline con lágrimas en los ojos & 17 & his eyes with his hand & 12 \\
\hline el pañuelo a los ojos & 17 & in the eyes of the & 12 \\
\hline en un abrir y cerrar de ojos & 17 & with his eyes fixed on the & 12 \\
\hline la mano por los ojos & 17 & her handkerchief to her eyes & 11 \\
\hline los ojos fijos en el & 17 & raising his eyes to the & 11 \\
\hline un abrir y cerrar de ojos & 17 & with tears in his eyes & 11 \\
\hline un ojo de la cara & 17 & & \\
\hline la mano a los ojos & 16 & & \\
\hline las niñas de sus ojos & 14 & & \\
\hline
\end{tabular}




\begin{tabular}{|l|c|l|l|}
\hline CorBPG & Frec. & CorCD & Frec. \\
\hline los ojos en el suelo & 14 & & \\
\hline los ojos en toda la & 13 & & \\
\hline los ojos llenos de lágrimas & 12 & & \\
\hline no quitaba los ojos de & 12 & & \\
\hline ojos fijos en el suelo & 12 & & \\
\hline a las niñas de sus ojos & 11 & & \\
\hline con los ojos fijos en el & 11 & & \\
\hline con los ojos llenos de & 11 & & \\
\hline los ojos en toda la noche & 11 & & \\
\hline los ojos fijos en el suelo & 11 & & \\
\hline ojos en toda la noche & 11 & & \\
\hline a los ojos de la & 10 & & \\
\hline
\end{tabular}

Los ejemplos identificados en ambos corpus con la palabra "mano" y hand, en segundo lugar, se muestran en la tabla 4:

Tabla 4. Clusters que contienen la palabra hand o "mano".

\begin{tabular}{|l|c|l|c|}
\hline CorBPG & Frec. & CorCD & Frec. \\
\hline la palma de la mano & 44 & his hands in his pockets & 77 \\
\hline con las manos en la & 43 & with his hands in his & 51 \\
\hline las manos en los bolsillos & 37 & with his hands in his pockets & 46 \\
\hline las palmas de las manos & 32 & hands in his pockets and & 36 \\
\hline llevándose las manos a la & 29 & his hands in his pockets and & 36 \\
\hline con las manos en los & 27 & with his hands to his & 27 \\
\hline con las manos en los bolsillos & 26 & with his hands in his pockets and & 21 \\
\hline de la mano de Dios & 24 & laying his hand upon his & 20 \\
\hline poniéndole la mano en el & 22 & his hands into his pockets & 17 \\
\hline de manos a boca con & 21 & with his hands behind him & 17 \\
\hline en la palma de la mano & 19 & his head upon his hand & 16 \\
\hline la mano en el pecho & 19 & his hand to his forehead & 15 \\
\hline la mano por la frente & 19 & the back of his hand & 14 \\
\hline
\end{tabular}




\begin{tabular}{|c|c|c|c|}
\hline CorBPG & Frec. & CorCD & Frec. \\
\hline se llevó las manos a & 18 & the palm of his hand & 14 \\
\hline puso la mano en el & 17 & hands into his pockets and & 13 \\
\hline con las manos a la & 16 & his hands into his pockets and & 13 \\
\hline el cielo con las manos & 16 & his hat in his hand & 13 \\
\hline le puso la mano en & 15 & laid his hand upon his & 13 \\
\hline yo me lavo las manos & 15 & laid his hand upon the & 13 \\
\hline llevó las manos a la & 14 & his hand as if he & 12 \\
\hline se llevó las manos a la & 14 & his hand in his pocket & 12 \\
\hline la mano por la cara & 13 & his hand upon the lock & 12 \\
\hline la mano en el bolsillo & 12 & put his hand to his & 12 \\
\hline pasó la mano por la & 12 & hands in his pockets and his & 11 \\
\hline poniéndole la mano en la & 12 & he laid his hand upon & 11 \\
\hline con la palma de la mano & 11 & his hands in his pockets and his & 11 \\
\hline dejado de la mano de & 11 & put his hands in his & 11 \\
\hline dejados de la mano de & 11 & the palms of his hands & 11 \\
\hline dejados de la mano de Dios & 11 & with his hand upon the & 11 \\
\hline en las palmas de las manos & 11 & her hands before her face & 10 \\
\hline la cara y las manos & 11 & him by the hand and & 10 \\
\hline nos tenga de su mano & 11 & his face with his hands & 10 \\
\hline dejado de la mano de Dios & 10 & his hands as if he & 10 \\
\hline Dios nos tenga de su mano & 10 & put his hands in his pockets & 10 \\
\hline el rostro con las manos & 10 & putting his hands in his & 10 \\
\hline el tío mano de mortero & 10 & putting his hands in his pockets & 10 \\
\hline la mano por el lomo & 10 & with his hand to his forehead & 10 \\
\hline le puso la mano en el & 10 & & \\
\hline se llevó la mano a & 10 & & \\
\hline
\end{tabular}


En la tabla 5, en tercer lugar, aparecen los ejemplos localizados en CorCD y CorBPG que contienen la palabra "cabeza" y head:

Tabla 5. Clusters que contienen la palabra head o "cabeza".

\begin{tabular}{|c|c|c|c|}
\hline CorBPG & Frec. & CorCD & Frec. \\
\hline las manos a la cabeza & 56 & his head on one side & 30 \\
\hline las manos en la cabeza & 39 & at the head of the & 27 \\
\hline con las manos en la cabeza & 35 & head on one side and & 19 \\
\hline llevándose las manos a la cabeza & 28 & the crown of his head & 17 \\
\hline la cabeza sobre el pecho & 19 & his head as if he & 16 \\
\hline la cabeza la idea de & 18 & his head upon his hand & 16 \\
\hline metido en la cabeza que & 18 & her head on one side & 14 \\
\hline ha metido en la cabeza & 17 & with his head on one & 14 \\
\hline la cabeza entre las manos & 14 & with his head on one side & 14 \\
\hline ha metido en la cabeza que & 13 & from head to foot and & 13 \\
\hline la calle de la cabeza & 13 & head from side to side & 13 \\
\hline los trastos a la cabeza & 13 & his head on one side and & 13 \\
\hline llevó las manos a la cabeza & 12 & his head out of the & 13 \\
\hline manos a la cabeza y & 12 & the back of his head & 13 \\
\hline que sí con la cabeza & 12 & trembling from head to foot & 13 \\
\hline se llevó las manos a la cabeza & 12 & head with an air of & 12 \\
\hline la cabeza en señal de & 11 & his head against the wall & 12 \\
\hline cabeza la idea de que & 10 & a shake of the head & 11 \\
\hline caer la cabeza sobre el & 10 & from head to foot with & 11 \\
\hline ha puesto en la cabeza & 10 & as he shook his head & 10 \\
\hline la mano por la cabeza & 10 & he shook his head and & 10 \\
\hline las manos a la cabeza y & 10 & him from head to foot & 10 \\
\hline más remedio que bajar la cabeza & 10 & his head from side to & 10 \\
\hline me quita de la cabeza & 10 & his head from side to side & 10 \\
\hline nadie me quita de la cabeza & 10 & it came into my head & 10 \\
\hline \multirow[t]{2}{*}{ remedio que bajar la cabeza } & 10 & shaking his head with a & 10 \\
\hline & & shook his head and said & 10 \\
\hline
\end{tabular}


Por último, los ejemplos que contienen la palabra "hombro" y shoulder aparecen detallados en la tabla 6:

Tabla 6. Clusters que contienen la palabra shoulder u "hombro".

\begin{tabular}{|l|c|l|c|}
\hline CorBPG & Frec. & CorCD & Frec. \\
\hline la mano en el hombro & 46 & hand upon his shoulder and & 11 \\
\hline poniéndole la mano en el hombro & 17 & her hand upon his shoulder & 11 \\
\hline puso la mano en el hombro & 13 & clapping him on the shoulder & 10 \\
\hline le puso la mano en el hombro & 10 & his hand upon his shoulder & 10 \\
\hline
\end{tabular}

De los resultados que se acaban de mostrar cabe destacar, en primer lugar, la amplia variedad de usos de lenguaje gestual empleados por ambos autores, si bien es cierto que algunos de los clusters que WordSmith genera son en realidad distintas realizaciones textuales de una misma acción ${ }^{10}$. Asimismo, conviene incidir en que existen algunos casos que no hacen referencia a ejemplos de lenguaje gestual. Estos casos tienen que ver con un uso figurado de la parte del cuerpo correspondiente (la niña de sus ojos, por ejemplo) o con ejemplos que en realidad corresponden al discurso de los personajes (it came into my head o Dios nos tenga de su mano, entre otros). Tales ejemplos, sin embargo, apenas comportan unos pocos casos aislados. Así, la mayoría de construcciones son empleadas por ambos autores para construir sus universos ficticios a través de la voz del narrador. Muchas de ellas, además, son similares en CorCD y CorBPG. En tales casos, como se podrá ver a continuación, parece advertirse una clara reproducción del estilo dickensiano por parte de Galdós.

\section{Análisis}

El análisis se ha dividido en cuatro bloques, uno por cada parte del cuerpo incluida en el estudio. En cada bloque se comparan los resultados obtenidos en ambos corpus y se analizan ejemplos concretos que servirán para ilustrar las concomitancias estilísticas entre Dickens y Galdós en lo que al uso del lenguaje gestual se refiere.

\section{1. "Ojo" - eye}

En el caso de los clusters localizados con las palabras “ojo" y eye, en primer lugar, se advierten usos muy similares en la producción de ambos autores. Como sostiene Korte (1997: 57), las miradas constituyen un componente fundamental del lenguaje gestual

10 Tómese como ejemplo el caso de his hands in his pockets, with his hands in his pockets, his hands in his pockets and, with his hands in his pockets and, hands in his pockets and his, his hands in his pockets and his, put his hands in his pockets y putting his hands in his pockets en la tabla 4. Todos comparten hands in his pockets, siendo ejemplos prácticamente idénticos de la realización textual de una misma acción. Si bien podrían haberse eliminado aquellos ejemplos que comportaran repeticiones de la misma secuencia gestual, se ha optado por mantener todos los resultados por una cuestión de transparencia, de tal suerte que aquellos lectores interesados en el material localizado puedan acceder a él sin necesidad de llevar a cabo el proceso metodológico detallado en el apartado 3. 
en el género novelesco. Estas han sido analizadas en la novela victoriana (Busse, 2010) y, también desde una perspectiva de corpus, en el estilo dickensiano (véase Mahlberg, 2013: 114-117). Precisamente en el análisis de las miradas en la producción de Dickens se hace hincapié, como no podía ser de otra manera, en el cluster más recurrente: his eyes fixed on the. Este cluster es, precisamente, también el más numeroso en las novelas de Galdós: con los ojos fijos en. En el uso que los autores hacen de ambos, además, se advierte un valor parecido. Como señala Mahlberg (2013: 116), la fijación de la mirada de un personaje es empleada por Dickens para reforzar la actitud de este, como se puede observar en (3). En la producción de Galdós, como se puede comprobar en (4), la construcción con los ojos fijos en desempeña una función similar:

(3) Herbert said of himself, with his eyes fixed on the fire, that he thought he must have committed a felony and forgotten the details of it, he felt so dejected and guilty.

(Great Expectations, capítulo 36)

(4) La Nela no decía nada. Después de mostrar exaltada alegría, meditaba con los ojos fijos en el suelo.

(Marianela, capítulo 8)

Sin embargo, la concomitancia estilística que existe entre ambos autores en el uso de clusters que contienen la palabra eye u "ojo" va más allá de este ejemplo. Como se puede observar en la tabla 3, aparte de his eyes fixed on the y de con los ojos fijos en, en ambos corpus se han localizado varios ejemplos casi idénticos. Tal es el caso de his eyes with his hand y la mano a los ojos, with tears in his eyes y con lágrimas en los ojos o her handkerchief to her eyes y el pañuelo a los ojos. Tanto with tears in his eyes y con lágrimas en los ojos, por un lado, como her handkerchief to her eyes y el pañuelo a los ojos, por otro, resultan casos especialmente interesantes, pues en ellos se advierte un uso sorprendentemente similar por parte de ambos novelistas. With tears in his eyes y con lágrimas en los ojos, por una parte, son empleados por Dickens y Galdós para expresar la tristeza de personajes de sexo masculino. En el caso de Dickens, la asociación del cluster a personajes masculinos está clara, pues el adjetivo his así lo revela. He aquí dos ejemplos:

(5) 'Good gracious, Misses Brown, no!' returned the Grinder, with tears in his eyes. 'Was there ever such a-!'

(Dombey and Son, capítulo 52)

(6) 'And if we were no better off than anybody else, what would become of our sense of gratitude; which,' said Mr Pecksniff with tears in his eyes, as he shook his fist at a beggar who wanted to get up behind, 'is one of the holiest feelings of our common nature.'

(Martin Chuzzlewit, capítulo 8)

En el caso de Galdós, de los diecisiete ejemplos localizados de con lágrimas en los ojos, hasta catorce se asocian a la tristeza de hombres. Por ejemplo:

(7) ‘Sabes lo que me ofrecen por mi resellamiento?’ — añadió Bravo casi con lágrimas en los ojos_- 'Pues la secretaría de un Gobierno de provincia, o un destino en Cuba, a elegir.' 
(8) '¡Oh! Pasaron aquellos tiempos de gloria’ —exclamó D. Patricio con lágrimas en los ojos y declamando con cierto énfasis que no cuadraba mal a su hueca voz y alta figura.

(El terror de 1824, capítulo 1)

Esta similitud en el uso de with tears in his eyes y con lágrimas en los ojos no se detiene aquí, pues Dickens y Galdós también hacen un uso parecido de ellos desde un punto de vista formal. Así, como se puede advertir en los ejemplos que acaban de mostrarse, ambos autores tienden a emplear estos clusters en momentos dialógicos. Esto es, las intervenciones de los personajes, que revelan la tristeza de estos, se ven reforzadas por el lenguaje gestual, que sirve para ilustrar sus sentimientos. Esta es una idiosincrasia dickensiana, empleada por el autor victoriano para lograr efectos de autenticidad a través de la simultaneidad del habla de los personajes y la comunicación no verbal (Mahlberg, Smith y Preston, 2013: 40). A la luz de los ejemplos localizados en la producción de Galdós, parece claro que el autor canario pudo imitar esta estrategia para realzar la tristeza de varios de sus personajes masculinos en sus novelas.

Por otra parte, en la utilización de her handkerchief to her eyes y el pañuelo a los ojos se observa un uso similar al de with tears in his eyes y con lágrimas en los ojos, pues her handkerchief to her eyes es empleado por Dickens en exclusiva con personajes femeninos y el pañuelo a los ojos es empleado por Galdós también mayormente con mujeres. En el caso de Dickens, la asociación de her handkerchief to her eyes con mujeres resulta nuevamente clara, pues her revela el sexo femenino de los personajes con los que se asocia. Por ejemplo:

(9) 'I fear he was,' said Mrs Nickleby, with her handkerchief to her eyes. 'If it hadn't been for me, I don't know what would have become of him.'

(Nicholas Nickleby, capítulo 10)

(10) 'Oh Kit!'said his mother, with her handkerchief to her eyes, 'what have you done! I never can go there again-never!'

(The Old Curiosity Shop, capítulo 41)

En el caso de Galdós, aunque algunos ejemplos se emplean refiriéndose a hombres, más del sesenta por ciento (once de los diecisiete ejemplos localizados), como se decía, son empleados con mujeres. Por ejemplo:

(11) Presentacioncita se sintió conmovida, y llevándose el pañuelo a los ojos, dijo: -Está preso.

(Memoria de un cortesano de 1815, capítulo 9)

(12) Irene se llevó el pañuelo a los ojos, y con voz de ahogo me dijo: 'Sabe usted... más que Dios...'

(El amigo Manso, capítulo 41)

Desde luego, resulta muy elocuente que tanto Dickens como Galdós no solo establezcan una distinción entre personajes de distinto sexo para describir la tristeza, sino que, además, ambos recurran a construcciones similares para proyectar la de hombres por un lado y la de mujeres por otro. Asimismo, en el caso de her handkerchief to her eyes y el pañuelo a los ojos cabe destacar nuevamente su empleo en momentos conversacionales, tal y como se puede observar en los ejemplos (9) a (12). 
Al igual que el de los hombres, el discurso de las mujeres del universo galdosiano en momentos de tristeza se ve reforzado, en un estilo que podríamos denominar dickensiano, por un lenguaje gestual muy específico que contribuye a reforzar la desazón de estas a través del pañuelo que se llevan a los ojos cuando hablan.

En suma, las miradas de los personajes de las novelas de Dickens y Galdós parecen revelar un eco dickensiano en el estilo del autor canario hasta ahora desapercibido. Así, no solo en la actitud que deja entrever la fijación de las miradas de los personajes - verbalizada a través de construcciones que resultan ser las más numerosas en ambos corpus-, sino también en la suerte de dicotomía que los dos novelistas establecen a la hora de proyectar la tristeza de hombres y mujeres, se establece un paralelismo en el estilo de ambos autores que refuerza la influencia del autor victoriano en Galdós desde un punto de vista estilístico.

\section{2. "Mano" - hand}

En los clusters localizados con las palabras "mano" y hand, en segundo lugar, también se han identificado paralelismos dignos de análisis. Como se puede observar en la tabla 4, se trata de la parte del cuerpo con la que se han generado más clusters en ambos corpus. Como en el caso de aquellos que contienen la palabra eye u "ojo" que acaban de analizarse, también existen ejemplos muy parecidos en ambos corpus. Tal es el caso de the palm of his hands y la palma de la mano, his hand to his forehead y la mano por la cara, his face with his hand y el rostro con las manos o his hands in his pockets y las manos en los bolsillos. Esta última pareja, debido sobre todo a la sistematicidad de his hands in his pockets en CorCD, resulta especialmente interesante. Como se puede advertir en la tabla 4 , his hands in his pockets es el cluster más numeroso de todos los que se han generado en el corpus de Dickens, con setenta y siete apariciones. Esta construcción constituye un rasgo característico del estilo del autor victoriano (Mahlberg, 2013: 65). Dickens la emplea en cada una de sus catorce novelas para describir la posición de los hombres - y no la de mujeres- dentro de la escena. Al contrario que los clusters analizados anteriormente, este no describe una sola actitud, sino que actúa como un bloque textual al que Dickens recurre de forma sistemática para construir su universo ficticio. Normalmente, los ejemplos suelen reforzar el carácter pensativo de los personajes con los que se asocia (13), de la misma manera que también es empleado cuando un personaje irrumpe en la escena y el narrador lo describe minuciosamente (14). Por ejemplo:

(13) 'Ah!' He began to walk about the room with his hands in his pockets, showing that he had been thinking as much.

(Bleak House, capítulo 50)

(14) Before Mr. Bounderby could reply, a young man appeared at the door, and introducing himself with the words, 'By your leaves, gentlemen!' walked in with his hands in his pockets. His face, close-shaven, thin, and sallow, was shaded by a great quantity of dark hair, brushed into a roll all round his head, and parted up the centre.

(Hard Times, libro 1, capítulo 6)

Estos usos de his hands in his pockets gozan nuevamente de réplica en la obra de Galdós, que emplea las manos en los bolsillos de una forma similar. Así, son los 
hombres y no las mujeres quienes aparecen presentados en esta pose y, también como en Dickens, se trata de un bloque textual empleado por el autor para modelar su universo ficticio. Además, resulta sorprendente que, como el autor inglés, Galdós lo utilice con fines similares. Así, las manos en los bolsillos también es empleado habitualmente para reforzar el estado meditabundo de un personaje o para introducirlo antes de describirlo de forma detallada, como se puede observar en los ejemplos (15) y (16) respectivamente:

(15) Ambos le miraron. Miquis se paseaba a lo largo de la sala, con las manos en los bolsillos, arrastrando sus miradas por el suelo.

(El doctor Centeno, capítulo 3)

(16) Delante de la miserable casilla que le servía de alojamiento solía pasearse D. Francisco por las tardes con las manos en los bolsillos de su capote, y pisando fuerte para que entraran en calor las entumecidas piernas. Era hombre de cuarenta y dos años, recio y avellanado, de semblante rudo, en que se pintaba una gran energía, y todo su aspecto revelaba al guerreador castellano, más ágil que forzudo.

(Los Cien Mil Hijos de San Luis, capítulo 8)

Este paralelismo se ve reforzado si tenemos en cuenta que ambos autores, además, emplean his hands in his pockets y las manos en los bolsillos con los verbos walk y "pasear" respectivamente, tal y como se puede observar en los ejemplos anteriores. Así, a los personajes no solo se les describe con las manos en los bolsillos en ambos casos, sino que además caminan, lo que ayuda a proyectar efectos como el mencionado carácter reflexivo. Este componente de dinamismo que llevan aparejados, unido al hecho de que se empleen para dar cuenta de los mismos aspectos y que sean los hombres y no las mujeres con quienes se asocian, hacen de his hands in his pockets y las manos en los bolsillos otro ejemplo más en el que se advierte un eco dickensiano en la producción de Galdós en lo que al lenguaje gestual se refiere.

\section{3. "Cabeza" - head}

En el caso de los clusters generados en CorBPG y CorCD con las palabras "cabeza" y head respectivamente, en tercer lugar, también se advierten concomitancias estilísticas dignas de mención. Cabe destacar que la cabeza desempeña un papel importante en la producción de Galdós como recurso de caracterización por sí misma. Así lo demuestran ejemplos como que sí con la cabeza. De los doce usos localizados en el corpus (ver tabla 5), seis se asocian a un mismo personaje: Fortunata (Fortunata y Jacinta). El uso repetido de esta construcción - se emplea con este personaje tantas veces como con el resto de figuras de la totalidad de sus novelas - sirve para reforzar el carácter sumiso de la joven, tal y como se puede advertir en los siguientes dos ejemplos:

(17) Fortunata dijo que sí con la cabeza, y el dogal que en el cuello sentía empezó a aflojarse.

(Fortunata y Jacinta, parte 3, capítulo 6)

(18) Fortunata dijo que sí con la cabeza, y sus miradas daban a entender que aquel perdón era de los fáciles, porque el amor andaba de por medio.

(Fortunata y Jacinta, parte 4, capítulo 6) 
Sin embargo, más allá del valor estilístico de este cluster como recurso de caracterización, en el corpus de Galdós también se advierte un componente dickensiano digno de reseña. Como se puede observar en la tabla 5, ambos autores emplean de forma recurrente una construcción similar: his head upon his hand y la cabeza entre las manos. Tanto Dickens como Galdós la utilizan fundamentalmente para ilustrar la tristeza de sus personajes. Sirvan como ejemplo, en primer lugar, los siguientes dos ejemplos extraídos de CorCD:

(19) I was so confounded by the alteration in him, that at first I could only observe him in silence, as he stood leaning his head upon his hand, and looking gloomily down at the fire.

(David Copperfield, capítulo 22)

(20) He appeared so miserable, sitting with his head upon his hand, and I appeared so unwelcome, that I was going to offer an apology when Mr. Bucket took the matter on himself.

(Bleak House, capítulo 59)

Efectivamente, Dickens construye el carácter desdichado de sus personajes, entre otros aspectos, a través de este gesto tan particular, que es replicado por Galdós en sus novelas. Por ejemplo:

(21) Junto a ella, y sentada en el suelo, con la cabeza entre las manos y apoyada en el lecho, estaba Inés. Su llanto tranquilo era el natural desahogo de un dolor resignado, propio de quien acostumbraba a relacionar las penas y las alegrías con la voluntad de arriba.

(La Corte de Carlos IV, capítulo 28)

(22) Nomdedeu, sin atreverse a contradecirla habíase sentado junto a ella, y con la cabeza entre las manos lloraba como un chiquillo.

(Gerona, capítulo 5)

Como se puede advertir, el llanto es empleado en (21) y (22) como elemento principal sobre el que se sustenta la tristeza de los personajes. Sin embargo, este se refuerza a través del gesto de colocar la cabeza entre las manos por parte de ambos. Llama la atención, además, que tanto Inés (21) como el doctor Nomdedeu (22) realicen este gesto mientras se encuentran sentados, lo que hace de la cabeza entre las manos un bloque textual aún más sólido y refuerza el hecho de que Galdós lo empleara, de un modo similar a Dickens, como recurso para proyectar desdicha entre sus personajes. La utilización de estas construcciones parecidas por parte de ambos autores para expresar las mismas emociones parece constituir, pues, otro ejemplo más que revela el componente dickensiano en la obra de Galdós a la hora de construir su universo ficticio a través del lenguaje gestual de sus personajes.

\section{4. "Hombro" - shoulder}

Los clusters identificados con las palabras "hombro" y shoulder, por último, son igualmente representativos. En ambos corpus se han identificado construcciones muy parecidas que, además, también desempeñan una función similar. Tal es el caso de her hand upon his shoulder y his hand upon his shoulder en CorCD y de ponién- 
dole la mano en el hombro y le puso la mano en el hombro en CorBPG. Dickens, por un lado, emplea her hand upon his shoulder y his hand upon his shoulder para reforzar el afecto de un personaje hacia otro (Mahlberg, 2013: 117). Tómense como ejemplo los siguientes dos usos de her hand upon his shoulder:

(23) 'Oh, I am not unhappy, cousin John!'said Ada, smiling cheerfully, with her hand upon his shoulder, where she had put it in bidding him good night. 'But I should be a little so if you thought at all the worse of Richard.'

(Bleak House, capítulo 17)

(24) 'I think, my dear son, 'returned Mrs. Maylie, laying her hand upon his shoulder, 'that youth has many generous impulses which do not last [...].'

(Oliver Twist, capítulo 34)

Este uso se ve replicado en la producción de Galdós en su empleo de poniéndole la mano en el hombro y le puso la mano en el hombro, como se puede advertir en los siguientes ejemplos de poniéndole la mano en el hombro:

(25) 'Querida tía' —indicó poniéndole la mano en el hombro —. 'Si me contesta Vd. con lágrimas y suspiros, me conmoverá pero no me convencerá.'

(Doña Perfecta, capítulo 19)

(26) 'Hijo de mi alma — dijo Fidela gozosa, poniéndole la mano en el hombro-. 'Mecenas quiere decir: protector de las letras.'

(Torquemada en el Purgatorio, parte 1, capítulo 10)

Como se puede comprobar, Galdós también utiliza esta construcción para expresar el afecto de un personaje hacia otro. El eco dickensiano en el uso que Galdós hace de este cluster se refuerza si tenemos en cuenta que lo emplea, al igual que Dickens, para acompañar el discurso del personaje que pone la mano en el hombro del otro. Además, cabe destacar que el autor canario hace un uso similar de estas construcciones desde un punto de vista formal. Como se puede observar en los ejemplos anteriores, tanto Dickens como Galdós las emplean en una interrupción del discurso de los personajes. Este es un estilema típicamente dickensiano, conocido como "suspended quotation" (Lambert, 1981), que Galdós parece imitar en sus novelas. A ello hay que añadir que en todos los ejemplos se observa un mismo patrón: (i) el discurso del personaje que precede la interrupción del narrador en la que se inserta el cluster contiene un vocativo con el que el personaje se refiere directamente a la persona que goza de su afecto; (ii) este vocativo es interrumpido por el comentario del narrador, que se refuerza a través del uso del cluster; (iii) una vez descrito el lenguaje gestual - en términos similares por parte de ambos autores-, el narrador continúa con la reproducción del discurso del personaje. En definitiva, tanto el parecido de los clusters en cuestión, como la función similar que desempeñan en la producción de ambos autores y el uso prácticamente idéntico que ambos hacen de ellos desde un punto de vista estructural, revelan otro componente dickensiano en la producción de Galdós en lo que a la utilización del lenguaje gestual se refiere.

A la luz de los ejemplos que se han desgranado con cada una de las partes del cuerpo que conforman el estudio, parece claro que la influencia que Dickens ejerció sobre Galdós tiene un carácter más profundo del tradicionalmente apuntado por la crítica. Naturalmente, los ejemplos a los que se suele hacer alusión - la mayoría de 
carácter impresionista, basados en situaciones y sobre todo en personajes en los que se advierte un paralelismo con Dickens - son una muestra clara de la devoción que Galdós sentía por el autor inglés, de la misma manera que algunas técnicas como la simbología de la onomástica o las muletillas empleadas por los personajes son un rastro evidente del estilo del novelista victoriano. Sin embargo, como cabría esperar, esta influencia se cifra también en aspectos más sutiles, como los ejemplos en relación al lenguaje gestual que acaban de analizarse han demostrado. Es cierto que afirmar que Galdós empleara deliberadamente un cluster determinado por imitación directa de Dickens puede resultar un tanto atrevido. No obstante, en virtud de la gran cantidad de ejemplos similares localizados en ambos corpus, puede concluirse que el lenguaje gestual empleado por Galdós ofrece tintes dickensianos y que este, sobre todo teniendo en cuenta el carácter prácticamente análogo de los casos que aquí se han analizado y sus similitudes tanto en términos formales como funcionales, parece ser fruto de la influencia que sobre él ejerció el novelista inglés.

\section{Apuntes finales}

Los estudios de estilística de corpus han permitido analizar cuestiones de estilo en el campo de la literatura desde perspectivas tradicionalmente inabarcables. La eficacia de estos enfoques contrasta, sin embargo, con la ausencia de estudios de este tipo sobre autores en lengua española. En este sentido, podría decirse que el presente artículo ha pretendido demostrar el potencial de los enfoques de corpus en el análisis de autores en español. De forma más precisa, el estudio ha girado en torno a la consabida influencia que Charles Dickens ejerció sobre Benito Pérez Galdós. Esta influencia, que tradicionalmente se ha sustentado en referencias de tipo impresionista más que en estudio sistemáticos de la lengua de ambos, ha sido abordada desde un punto de vista puramente estilístico. El análisis se ha centrado en el lenguaje gestual y, más concretamente, en segmentos de texto que contienen referencias a partes del cuerpo - los ojos, la cabeza, las manos y los hombros - cuya relevancia estilística en las novelas de Dickens ya ha sido demostrada con anterioridad. Estas partes del cuerpo han servido de punto de partida desde el que identificar los clusters que se han examinado. El hecho de que el análisis se haya centrado en construcciones generadas de forma automática despeja cualquier indicio de tendenciosidad y sin duda refuerza las conclusiones que se han extraído. En concreto, a través del análisis de los segmentos de textos identificados se ha podido demostrar cómo ambos autores recurren a construcciones similares para modelar sus universos ficticios e ilustrar las mismas emociones en sus personajes, lo que parece reflejar un eco dickensiano en el estilo de Galdós.

Por último, cabe destacar que el enfoque de corpus que se ha adoptado ha permitido localizar una gran cantidad de material que, aunque no se haya analizado de forma detenida en este estudio, resulta un filón desde el punto de vista de la exégesis del autor canario. Tal es el caso de construcciones como la comentada que sí con la cabeza, empleada eminentemente por Galdós para describir a Fortunata en Fortunata y Jacinta. Este ejemplo tiene un valor caracterizador claro, comparable al de otros rasgos idiosincrásicos del personaje como su uso sistemático de la interjección "quia". La localización de este tipo de construcciones, así como su cuantificación y su distribución en la producción literaria del autor, solo resulta posible gracias a 
una aproximación de corpus, lo que demuestra el potencial de este tipo de enfoques con autores en lengua española en general y descubre nuevas vías de análisis en los estudios galdosianos en particular.

\section{Obras citadas}

Adolphs, Svenja, y Ronald Carter, "Corpus stylistics: point of view and semantic prosodies in To the Lighthouse", Poetica, 58 (2002), pp. 7-20.

Barnbrook, Geoff, Language and Computers. Edinburgh, Edinburgh University Press, 1996.

Benítez, Rubén, Cervantes en Galdós, Murcia, Universidad de Murcia, 1990.

Berglund, Ylva, Alan Morrison, Rowan Wilson y Martin Wynne, An Investigation into Free Ebooks, 2004. Disponible en: https://ota.ox.ac.uk/documents/ebooks/report/FreeEbooks. html (consultado por última vez el 31 de julio de 2017).

Busse, Beatrix, Speech, Writing and Thought Presentation in a Corpus of Nineteenth-Century English Narrative Fiction, Bern, University of Bern, 2010.

Biblioteca Virtual Miguel de Cervantes. Disponible en: http://www.cervantesvirtual.com/ (consultado por última vez el 31 de julio de 2017).

Correa, Gustavo, Realidad, ficción y símbolo en las novelas de Pérez Galdós: ensayo de estética realista, Madrid, Gredos, 1967.

Culpeper, Jonathan, "Keyness: Words, parts-of-speech and semantic categories in the character-talk of Shakespeare's Romeo and Juliet", International Journal of Corpus Linguistics, 14.1 (2009), pp. 29-59. DOI: 10.1075/ijcl.14.1.03cul

Fischer-Starcke, Bettina, Corpus Linguistics in Literary Analysis: Jane Austen and her Contemporaries, London, Continuum, 2010.

Goldman, Peter, "Galdós and Cervantes: two articles and a fragment", Anales Galsianos, 4 (1971), pp. 99-106.

John, Juliet, Dickens's Villains: Melodrama, Character, Popular Culture, Oxford, Oxford University Press, 2001.

Korte, Barbara, Body Language in Literature, Toronto, University of Toronto Press, 1997.

Lacosta, Francisco C, "Galdós y Balzac”, Cuadernos Hispano-Americanos, 224/225 (1968), pp. 345-374.

Lambert, Michael, Dickens and the Suspended Quotation, New Haven, Yale University Press, 1981.

Levy, Melania, La influencia de Dickens en Galdós, New York, Columbia University, 1937.

Mahlberg, Michaela, "Clusters, key clusters and local textual functions in Dickens", Corpora, 2.1 (2007), pp. 1-31.

—, Corpus Stylistics and Dickens's Fiction, London, Routledge, 2013.

Mahlberg, Michaela, y Catherine Smith, "Dickens, the suspended quotation and the corpus", Language and Literature, 21.1 (2012), pp. 51-65. DOI: 10.1177/0963947011432058

Mahlberg, Michaela, Catherine Smith y Simon Preston, "Phrases in literary contexts: patterns and distributions of suspensions in Dickens's novels", International Journal of Corpus Linguistics, 18.1 (2013), pp. 35-56. DOI: 10.1075/ijcl.18.1.05mah

Manning, Chris, y Hinrich Schtüze, Foundations of Statistical Natural Language Processing, Cambridge, MIT Press, 1999.

McGovern, Timothy, Dickens in Galdós, New York, Peter Lang, 2000.

Ollero, Carlos, "Galdós y Balzac”, en Douglass M. Rogers (ed.), Benito Pérez Galdós. El escritor y la crítica, Madrid, Taurus, 1973, pp. 185-193. 
Pérez Galdós, Benito, Obras completas. Novelas. Tomo III, Madrid, Aguilar, 1980.

Proyecto Gutenberg. Disponible en: http://www.gutenberg.org/ (consultado por última vez el 31 de julio de 2017).

Römer, Ute, "Where the computer meets language, literature and pedagogy: Corpus analysis in English studies", en Andrea Gerbig y Anja Müller-Wood (eds.), How Globalization Affects the Teaching of English: Studying Culture through Texts, Lampeter, E. Mellen Press, 2006, pp. 81-109.

Ruano San Segundo, Pablo, “A corpus-stylistic approach to Dickens' use of speech verbs: Beyond mere reporting”, Language and Literature, 25.2 (2016), pp. 113-29. DOI: 10.1177/0963947016631859

—, "Charles Dickens's influence on Shaw revisited: A corpus-based stylistic study", SHAW: The Journal of Bernard Shaw Studies, 37.2 (2017), pp. 260-81.

- , "Charles Dickens's gender-based use of speech verbs: a stylistic analysis", Gender and Language, 12.2 (2018), pp. 192-217. DOI: 10.1558/genl.31137.

Scott, Mike, WordSmith Tools version 7, Stroud, Lexical Analysis Software, $2016 \mathrm{a}$.

—, WordSmith Tools Help, Stroud, Lexical Analysis Software, 2016b.

Stubbs, Michael, "Conrad in the computer: Examples of quantitative stylistics methods", Language and Literature, 14.1 (2005), pp. 5-24. DOI: 10.1177/0963947005048873.

Tambling, Jeremy, "Dickens and Galdós", en Michael Hollington (ed.), The Reception of Charles Dickens in Europe, London, Bloomsbury, 2013, pp. 191-96.

Wright, Chad C., "Artifacts and effigies: the Porreño household revisited", Anales Galdosianos, 14 (1979), pp. 13-24. 